\title{
The Action of the Symmetric Group on a Generalized Partition Semilattice
}

\author{
Robert Gill \\ Department of Mathematics and Statistics \\ University of Minnesota Duluth \\ 10 University Drive \\ Duluth, MN 55812 \\ rgill@d.umn.edu
}

Submitted: February 7, 1998; Accepted: April 21, 2000

Key Words: hyperplane arrangement, Möbius function, homology, induced character, cyclic group

AMS Subject Classification (1991): Primary 06A07; Secondary 05E25, 52B30, 20C30, 11A05

\begin{abstract}
Given an integer $n \geq 2$, and a non-negative integer $k$, consider all affine hyperplanes in $\mathbb{R}^{n}$ of the form $x_{i}=x_{j}+r$ for $i, j \in[n]$ and a non-negative integer $r \leq k$. Let $\Pi_{n, k}$ be the poset whose elements are all nonempty intersections of these affine hyperplanes, ordered by reverse inclusion. It is noted that $\Pi_{n, 0}$ is isomorphic to the well-known partition lattice $\Pi_{n}$, and in this paper, we extend some of the results of $\Pi_{n}$ by Hanlon and Stanley to $\Pi_{n, k}$.

Just as there is an action of the symmetric group $\mathfrak{S}_{n}$ on $\Pi_{n}$, there is also an action on $\Pi_{n, k}$ which permutes the coordinates of each element. We consider the subposet $\Pi_{n, k}^{\sigma}$ of elements that are fixed by some $\sigma \in \mathfrak{S}_{n}$, and find its Möbius function $\mu_{\sigma}$, using the characteristic polynomial. This generalizes what Hanlon did in the case $k=0$. It then follows that $(-1)^{n-1} \mu_{\sigma}\left(\Pi_{n, k}^{\sigma}\right)$, as a function of $\sigma$, is the character of the action of $\mathfrak{S}_{n}$ on the homology of $\Pi_{n, k}$.

Let $\Psi_{n, k}$ be this character times the sign character. For $\mathfrak{C}_{n}$, the cyclic group generated by an $n$-cycle $\sigma$ of $\mathfrak{S}_{n}$, we take its irreducible characters and induce
\end{abstract}


them up to $\mathfrak{S}_{n}$. Stanley showed that $\Psi_{n, 0}$ is just the induced character $\chi \uparrow_{\mathfrak{C}_{n}}^{\mathfrak{S}_{n}}$ where $\chi(\sigma)=e^{2 \pi i / n}$. We generalize this by showing that for $k>0$, there exists a non-negative integer combination of the induced characters described here that equals $\Psi_{n, k}$, and we find explicit formulas. In addition, we show another way to prove that $\Psi_{n, k}$ is a character, without using homology, by proving that the derived coefficients of certain induced characters of $\mathfrak{S}_{n}$ are non-negative integers.

\section{Introduction}

Given a finite partially ordered set $P$, let $\leq$ denote the partial order, and assume that $P$ has a unique minimal element $\hat{0}$. An automorphism $\sigma$ on $P$ is a permutation of the elements of $P$ such that if $x \leq y$, then $\sigma(x) \leq \sigma(y)$. Let $P^{\sigma}$ be the subposet of $P$ that consists of the elements that are fixed by $\sigma$. If $P$ is a lattice, then so is $P^{\sigma}$ (For a proof, see page 319 of [9]).

Now we look at one particular lattice. For some positive integer $n$, if we let $\Pi_{n}$ denote the set of all partitions of the set $[n]=\{1,2, \ldots, n\}$, ordered by refinement, then $\Pi_{n}$ is a lattice. There has been a lot of work on $\Pi_{n}$, and the action of the symmetric group $\mathfrak{S}_{n}$ on it. An element of $\mathfrak{S}_{n}$ permutes the elements of $[n]=\{1,2, \ldots, n\}$, and therefore acts as an automorphism on $\Pi_{n}$. Given $\sigma \in \mathfrak{S}_{n}$, let $\Pi_{n}^{\sigma}$ denote the subposet of $\Pi_{n}$ of elements that are fixed by $\sigma$.

The Möbius function $\mu$ is defined on intervals $[x, y]=\{z: x \leq z \leq y\}$ of a poset $P$ such that $\mu(x, x)=1$ for all $x \in P$ and for $x<y$,

$$
\sum_{z \in[x, y]} \mu(x, z)=0
$$

If $P$ has $\hat{1}$, then define $\mu(P)$ to be $\mu(\hat{0}, \hat{1})$. Let $\mu_{\sigma}$ be the Möbius function of $\Pi_{n}^{\sigma}$. In 1981, Hanlon [9, Th. 4] showed that

$$
\mu_{\sigma}\left(\Pi_{n}^{\sigma}\right)= \begin{cases}\mu(n / d)\left(-\frac{n}{d}\right)^{d-1}(d-1) ! & \text { if } \sigma \text { is a product of } d \text { cycles } \\ 0 & \text { of length } n / d \text { for some } d \mid n ; \\ & \text { otherwise. }\end{cases}
$$

Here, $\mu(n / d)$ is the classical number-theoretic Möbius function. In 1982, Stanley [13] used this result and the Lefschetz Fixed Point Theorem (stated in section 3) to show that as a function of $\sigma,(-1)^{n-1} \mu_{\sigma}\left(\Pi_{n}^{\sigma}\right)$ is the character of a particular representation of $\mathfrak{S}_{n}$ (Refer to [12, Ch.1] for definitions), its action on the top-dimensional homology of $\Pi_{n}$, which we define in section 3 . Let $\sigma$ be an $n$-cycle in $\mathfrak{S}_{n}$ and let $\mathfrak{C}_{n}$ be the cyclic subgroup of $\mathfrak{S}_{n}$ generated by $\sigma$. Let $\chi$ be the (irreducible) character of $\mathfrak{C}_{n}$ such that $\chi(\sigma)=e^{2 \pi i / n}$. Stanley showed that the induced character (defined in $\left.[12, \S 1.12]\right) \chi \uparrow_{\mathfrak{C}_{n}}^{\mathfrak{S}_{n}}$ equals this homology character times the sign character, which we denote $\Psi_{n}$. It is appropriate to show that $\Psi_{n}$ is an induced character from the cyclic group of order $n$ since it is zero for all elements of $\mathfrak{S}_{n}$ that are not conjugate to any element of $\mathfrak{C}_{n}$. 
In this paper, we extend these results of $\Pi_{n}$ to a generalized partition semilattice, which we now define. We will call it $\Pi_{n, k}$. The partition lattice is isomorphic to a poset of subspaces of $\mathbb{R}^{n}$ for $n \geq 2$, ordered by reverse inclusion, whose elements are all intersections of the hyperplanes

$$
H_{i, j}=\left\{x \in \mathbb{R}^{n}: x_{i}=x_{j}\right\},
$$

for $i, j \in[n]$, with minimal element $\mathbb{R}^{n}$. In other words, if $i$ and $j$ are in the same block of a partition of $\Pi_{n}$, then the corresponding subspace of $\mathbb{R}^{n}$ is contained in $H_{i, j}$.

Now consider the affine hyperplanes

$$
H_{i, j, r}=\left\{x \in \mathbb{R}^{n}: x_{i}=x_{j}+r\right\} .
$$

For a non-negative integer $k$, let $\Pi_{n, k}$ be the poset whose elements are all nonempty intersections of the $H_{i, j, r}$ such that $r \in \mathbb{Z}$ and $|r| \leq k$. These sets of hyperplanes are known as the extended Catalan arrangements (See after Theorem 2.3 in [14]). The unique minimal element is again the whole space $\mathbb{R}^{n}$, but there is more than one maximal element if $k>0$. For an affine subspace $X \in \Pi_{n, k}$, its dimension $\operatorname{dim}(X)$ is equal to the dimension of the linear translation of $X$, the set $\{v-x: v \in X\}$ for a particular $x \in X$. So $X$ is maximal if and only if $\operatorname{dim}(X)=1$.

First, the characteristic polynomial of a poset $P$ of affine subspaces of $\mathbb{R}^{n}$ is given by

$$
\lambda_{P}(t)=\sum_{X \in P} \mu(\hat{0}, X) t^{\operatorname{dim}(X)}
$$

In section 2, we let $P=\Pi_{n, k}$ and consider $P^{\sigma}$, the subposet of $P$ fixed by some $\sigma \in \mathfrak{S}_{n}$. We use the characteristic polynomial of $P$ and the paper by Hanlon [9] to show that the Möbius function of this subposet, $\mu_{\sigma}\left(P^{\sigma}\right)$, is as stated in (4). Then in section 3 , we use a result from Stanley's paper [13] to show that the character of the representation of $\mathfrak{S}_{n}$ acting on the top homology of $P$ is $(-1)^{n-1} \mu_{\sigma}\left(P^{\sigma}\right)$.

Let $\Psi_{n, k}$ be this character times the sign character, so $\left.\Psi_{n, k}=(-1)^{d-1} \mu_{\sigma}(P)^{\sigma}\right)$. In sections 4 and 5 , we show that $\Psi_{n, k}$ can be expressed as a non-negative integer combination of the characters of $\mathfrak{S}_{n}$ that are induced from irreducible characters of $\mathfrak{C}_{n}$, as in (15). First, we show that the induced characters in this sum are a basis for all induced characters from $\mathfrak{C}_{n}$. Then the main result in section 4 is that $\Psi_{n, k}$ is a sum of induced characters from $\mathfrak{C}_{d}$ for each $d \mid n$. In section 5 , we find an explicit expression for $\Psi_{n, k}$ in terms of these induced characters, also proving some concepts from number theory which we use along the way.

In the last section, we prove separately that the coefficients are non-negative integers, using the formula derived in Lemma 11, which gives us a way to prove that $\Psi_{n, k}$ is a character without proving that it is a homology character.

There is a lot more one can do on the subject of $\Pi_{n, k}$. For example, Christos Athanasiadis in his Ph.D. thesis [1] used the Möbius Inversion Formula to find the 
characteristic polynomial of numerous affine hyperplane arrangements, including this one [1, Th. 5.1]. Also, Julie Kerr in her Ph.D. thesis [10] discusses the poset obtained by adding a unique maximal element to $\Pi_{n, k}$. Although it becomes a lattice, its characteristic polynomial does not in general factor linearly as it does for $\Pi_{n, k}$. But its top-dimensional homology is isomorphic to a direct sum of copies of the algebra $\mathbb{C} \mathfrak{S}_{n}$, known as the regular representation of $\mathfrak{S}_{n}$. There is also additional work on the poset $\Pi_{n, k}$ in [7].

\section{The Möbius Function of $\Pi_{n, k}^{\sigma}$}

We first state [1, Th. 5.1]. This generalizes the characteristic polynomial of the wellknown partition lattice, which is the case $k=0$.

Theorem 1. The characteristic polynomial of $\Pi_{n, k}$ is given by

$$
\lambda_{\Pi_{n, k}}(t)=t(t-n k-1)(t-n k-2) \ldots(t-n(k+1)+1) .
$$

We now extend some more results of the partition lattice $\Pi_{n}$ to $\Pi_{n, k}$, first from Hanlon's paper [9]. Given any poset $P$ with a unique minimal element $\hat{0}$, let $\operatorname{Max}(P)$ denote the set of maximal elements of $P$ and let

$$
\mu(P)=\sum_{x \in \operatorname{Max}(P)} \mu(\hat{0}, x) .
$$

Now let $P=\Pi_{n, k}$ and consider the action of the symmetric group $\mathfrak{S}_{n}$ on $P$, permuting the coordinates of the elements. We consider the subposet $P^{\sigma}$, which consists of the elements of $P$ that are fixed by a permutation $\sigma \in \mathfrak{S}_{n}$, meaning whenever $X \in P^{\sigma}$ and $X \subseteq H_{i, j, r}$, then $X \subseteq H_{\sigma(i), \sigma(j), r}$. Note that if $\varepsilon$ is the identity permutation, then $P^{\varepsilon}=P$.

Let $\mu_{\sigma}$ denote the Möbius function in $P^{\sigma}=\Pi_{n, k}^{\sigma}$. The goal in this section is to prove that

$$
\mu_{\sigma}\left(P^{\sigma}\right)= \begin{cases}\mu(n / d)\left(-\frac{n}{d}\right)^{d-1}\left(\begin{array}{c}
(k+1) d-1 \\
d-1
\end{array}\right)(d-1) ! & \text { if } \sigma \text { is a product of } d \text { cycles } \\
0 & \text { of length } n / d \text { for some } d \mid n \\
& \text { otherwise }\end{cases}
$$

This is the Möbius function of $P^{\sigma}$, defined as in (3). It generalizes Hanlon's result for $k=0$, stated in (1). If $\sigma, \tau \in \mathfrak{S}_{n}$, then one can verify the isomorphism $P^{\sigma} \cong P^{\tau \sigma \tau^{-1}}$. Hence, viewed as a function of $\sigma, \mu_{\sigma}\left(P^{\sigma}\right)$ is a class function on $\mathfrak{S}_{n}$. It is in fact, up to a sign, a character of $\mathfrak{S}_{n}$, as we will soon see.

In order to find $\mu_{\sigma}\left(P^{\sigma}\right)$, we find the sum of the Möbius functions of each maximal interval of $P^{\sigma}$. The methods we use here are in many cases very similar to those used by 
Hanlon, with a slightly different poset. We state a well-known theorem that we will use here. Suppose we are given a finite lattice $L=[\hat{0}, \hat{1}]$. For some $x \in L$, define $\operatorname{Comp}(x)$ to be the set of complements of $x$ in $L$, i.e., $\operatorname{Comp}(x)=\{y \in L: x \wedge y=\hat{0}$ and $x \vee y=\hat{1}\}$. Then Crapo's Complementation Theorem [5, Th. 4] says that for any $x \in L$,

$$
\mu(L)=\sum_{\substack{y, z \in \operatorname{Comp}(x) \\ y \leq z}} \mu(\hat{0}, y) \mu(z, \hat{1}),
$$

and if some element of $L$ has no complements, then $\mu(L)=0$.

So we need to show that $[\hat{0}, X]^{\sigma}$ is a lattice for each $X \in \operatorname{Max}\left(P^{\sigma}\right)$. By $[18$, Prop. 3.1], since every element of $P$ is an intersection of affine hyperplanes from a given set, it is a geometric semilattice. Thus each maximal interval $[\hat{0}, X]$ in $P$ is a lattice, and by the first paragraph of section $1,[\hat{0}, X]^{\sigma}$ is a lattice too. So Crapo's Theorem applies here. Now we determine which element we use in Equation (5).

For each $\sigma \in \mathfrak{S}_{n}$, it can be verified that

$$
\operatorname{Max}\left(P^{\sigma}\right)=\operatorname{Max}(P) \cap P^{\sigma},
$$

which is mentioned in the proof of $[10$, Th. 2.1]. For $\sigma$, let

$$
\sigma=\sigma_{1} \sigma_{2} \ldots \sigma_{d}
$$

be the decomposition of $\sigma$ into disjoint cycles. For $i=1, \ldots, d$, let $C_{i}$ be the support of the cycle $\sigma_{i}$, that is, the set of all numbers from the cycle $\sigma_{i}$. It will be convenient to extend Hanlon's definition of the hinge of $\Pi_{n}[9$, p. 324], the partition which puts each cycle of $\sigma$ into its own block. Here, we want to extend it to any $k \geq 0$, so that it is an intersection of affine hyperplanes. In $\Pi_{n, 0}$ the element that corresponds to the hinge of $\Pi_{n}$ is the intersection of all $H_{j, l}$ such that $j$ and $l$ are in the same $C_{i}$. So this will be the hinge of $\Pi_{n, 0}$. The following lemma shows that for any $k$, only certain hyperplanes in $\Pi_{n, k}$ can contain the hinge.

Lemma 2. Suppose two numbers $i$ and $j$ are in the same cycle of $\sigma$, and for some $Z \in P^{\sigma}, Z$ is contained in $H_{i, j, r}$ for some $r$. Then $r=0$.

Proof. Suppose $Z \in P^{\sigma}$ and $\sigma_{1}$ is one of the disjoint cycles of $\sigma$ as in (7), with length $m \geq 2$. Suppose without loss of generality that $i, j \in C_{1}$ and $Z \subseteq H_{i, j, r}$. Then there exists an $s$ such that $\sigma^{s}(i)=j$, so let $\tau=\sigma^{s}$. Then $Z \subseteq H_{i, \tau(i), r}$ and then $Z \subseteq H_{\tau^{\omega}(i), \tau^{\omega+1}(i), r}$, since for each integer $\omega, P^{\sigma} \subseteq P^{\sigma^{\omega}}$. This means for any $z \in$ $Z, z_{i}=z_{\tau^{l}(i)}+l r$, so $z_{i}=z_{\tau^{m}(i)}+m r=z_{i}+m r$, since $\tau^{m}$ fixes all elements of $C_{1}$. Therefore $r=0$ and $Z \subseteq H_{i, j}$.

This proves that no nontrivial extension of the hinge is possible for $\Pi_{n, k}$. So define the hinge $h^{\sigma}$ of $P^{\sigma}$ to be the intersection of all $H_{j, l}$ for which $j$ and $l$ are in the same 
cycle of $\sigma$. For an element $Y \in \Pi_{n, k}$, define $\pi(Y)$ to be the partition that corresponds to $Y$. In other words, if $Y \subseteq H_{i, j, r}$ for some $r$, then $i$ and $j$ are in the same block of $\pi(Y)$. Therefore, each $C_{i}$ is a block of $\pi\left(h^{\sigma}\right)$. Then $\operatorname{dim}\left(h^{\sigma}\right)$ is the number of blocks of $\pi\left(h^{\sigma}\right)$ and the number of cycles of $\sigma$. For example, if $\sigma=(1,2,3)(4,5)(6) \in \mathfrak{S}_{6}$, then $h^{\sigma}=H_{1,2} \cap H_{1,3} \cap H_{2,3} \cap H_{4,5}$ in $\mathbb{R}^{6}$, and $\operatorname{dim}\left(h^{\sigma}\right)=3$. Notice that by Lemma 2, $h^{\sigma} \leq X$ for all $X \in \operatorname{Max}\left(P^{\sigma}\right)$, and that $h^{\sigma}$ is the greatest lower bound of all the maximal elements of $P^{\sigma}$. This is the element that whose complements we will find in order to prove the main result of this section. Now we are ready to prove one case of (4).

Theorem 3. $\mu_{\sigma}\left(P^{\sigma}\right)=0$ unless all disjoint cycles of $\sigma$ have the same length.

Proof. We prove this by showing that for a given $X \in \operatorname{Max}\left(P^{\sigma}\right), h^{\sigma}$ has no complements in $[\hat{0}, X]^{\sigma}$. If $\sigma$ is an $n$-cycle, then all cycles of $\sigma$ have the same length, and we do not consider that here. Otherwise, given any two blocks $B_{1}$ and $B_{2}$ of $\pi\left(h^{\sigma}\right)$, a given element $Z \in P^{\sigma}$ is a complement of $h^{\sigma}$ in $[\hat{0}, X]^{\sigma}$ only if there exists one element from each of the two blocks, say $i \in B_{1}$ and $j \in B_{2}$, such that $Z \subseteq H_{i, j, r}$ for some $r$. We need to show that if any two blocks of $\pi\left(h^{\sigma}\right)$ are not the same size, or equivalently, if any two cycles are not the same length, then there is an element less than $Z$ that is not $\hat{0}$ and is also less than $h^{\sigma}$.

Suppose we pick out two cycles from $\sigma$ that have different lengths. We can assume that $\sigma_{1}=(1, \ldots, m)$ and $\sigma_{2}=(m+1, \ldots, m+b)$, as defined in $(7)$, and $m<b$. In order for $Z$ to be a complement of $h^{\sigma}$ in $[\hat{0}, X]^{\sigma}, Z$ must be contained in some $H_{1, j, r}$ for some $r$ and for $m+1 \leq j \leq m+b$. So assume without loss of generality that $Z \subseteq H_{1, m+1, r}$, so then $Z \subseteq H_{s, m+s, r}$ for all $s=1, \ldots, m$. Let $g=\operatorname{gcd}(m, b)$. Then $g<b$ and $Z \subseteq H_{m+1, m+g+1}$. Let $Y$ be the intersection of all hyperplanes $H_{s-g, s}$ for $m+g<s \leq m+b$. Then $Z \geq Y$ and $\pi(Y)$ is a refinement of $\pi\left(h^{\sigma}\right)$, so since by Lemma 2, only hyperplanes $H_{i, j}$ can contain $h^{\sigma}, h^{\sigma} \geq Y$ too.

Therefore, $h^{\sigma} \wedge Z \geq Y>\hat{0}$, so $Z$ is not a complement of $h^{\sigma}$ in $[\hat{0}, X]^{\sigma}$. Since we chose an arbitrary $X \in \operatorname{Max}\left(P^{\sigma}\right)$, we have proved that $h^{\sigma}$ has no complements in any $[\hat{0}, X]^{\sigma}$. Thus $\mu_{\sigma}(\hat{0}, X)=0$ for all $X \in \operatorname{Max}\left(P^{\sigma}\right)$ and therefore, $\mu_{\sigma}\left(P^{\sigma}\right)=0$ unless all cycles of $\sigma$ have the same length.

Now we will find $\mu_{\sigma}\left(P^{\sigma}\right)$ for the other case of $(4)$, if $\sigma$ is a product of $d$ cycles of length $n / d$. To do this, we may assume that

$$
\sigma=(1,2, \ldots, j)(j+1, \ldots, 2 j) \cdots(n-j+1, \ldots, n),
$$

where $j=n / d$. Again, for each $X \in \operatorname{Max}\left(P^{\sigma}\right)$, we use complements of the hinge $h^{\sigma}$ in $[\hat{0}, X]^{\sigma}$ and equation (5). If $C \in \operatorname{Comp}\left(h^{\sigma}\right)$ in $[\hat{0}, x]^{\sigma}$, then $C \nsubseteq H_{\omega_{1}, \omega_{2}, r}$ for any $\omega_{1}, \omega_{2} \in[j]$ and any $r$, and

$$
C \subseteq H_{1, s j+i_{s}, r_{s}}
$$


for $s=1,2, \ldots, d-1$, and $r_{s}$ and $i_{s} \in[j]$ that depend on $s$. Note that $\operatorname{dim}(C)=j$ for all such $C$, so no two complements are comparable to each other. This will be used later to simplify (5). We now state the other case that we will prove, but we need a few lemmas first. Many of the lemmas here are similar to parts of [9, Lemma 6].

Theorem 4. $\mu_{\sigma}\left(P^{\sigma}\right)=\mu(n / d)\left(-\frac{n}{d}\right)^{d-1}\left(\begin{array}{c}(k+1) d-1 \\ d-1\end{array}\right)(d-1)$ ! if $\sigma$ is a product of $d$ disjoint cycles of length $n / d$.

Lemma 5. Given $X \in \operatorname{Max}\left(P^{\sigma}\right)$, if $C \in \operatorname{Comp}\left(h^{\sigma}\right)$ in $[\hat{0}, X]^{\sigma}$, then $[C, X]^{\sigma} \cong \mathfrak{D}_{j}$, the lattice of divisors of $j$. Thus, $\mu_{\sigma}(C, X)=\mu(n / d)$.

Proof. For any point in an affine subspace from $[C, X]^{\sigma}$, whatever equality is in the coordinates $1,2, \ldots, j$, the same equality holds for corresponding coordinates from the other blocks of $\pi\left(h^{\sigma}\right)$, depending on $i_{s}$ in (8). At the bottom element $C$ of the interval $[C, X]^{\sigma}$, for any $i_{1}, i_{2} \in[j], C \nsubseteq H_{i_{1}, i_{2}, r}$ for any $r$. At the maximal element, $X \subseteq H_{i_{1}, i_{2}}$ by Lemma 2. So $[C, X]^{\sigma}$ here is isomorphic to $[C, \hat{1}]^{\sigma}$ in the case $k=0$. Since $[9$, Lemma $6 \mathrm{c}]$ says that $[C, \hat{1}]^{\sigma} \cong \mathfrak{D}_{n / d}$, we are done.

Lemma 6. There exists a one-to-one correspondence between the maximal elements of $P^{\sigma}$ and the maximal elements of $\Pi_{d, k}$.

Proof. If $d=1$, then $P^{\sigma}$ has only one maximal element, and $\left|\Pi_{1, k}\right|=1$. If $d \geq 2$, then by Lemma 2 , if $X \in \operatorname{Max}\left(P^{\sigma}\right)$, then $X \subseteq H_{j(i-1)+\omega, j(i-1)+\omega+1}$ for all $i=1, \ldots, d$ and all $\omega \in[j-1]$. Then the $X \in \operatorname{Max}\left(P^{\sigma}\right)$ such that $X \subseteq H_{j\left(\omega_{1}-1\right)+1, j\left(\omega_{2}-1\right)+1, r} \subseteq \mathbb{R}^{n}$ corresponds to the $Y \in \operatorname{Max}\left(\Pi_{d, k}\right)$ such that $Y \subseteq H_{\omega_{1}, \omega_{2}, r} \subseteq \mathbb{R}^{d}$, and vice-versa. So this correspondence is a bijection.

Lemma 7. Given a maximal element $X \in P^{\sigma}$, let $Y$ be its corresponding maximal element in $\Pi_{d, k}$, as described in Lemma 6 . If $d \geq 2$, then for all $C \in \operatorname{Comp}\left(h^{\sigma}\right)$ in $[\hat{0}, X]^{\sigma},[\hat{0}, C]^{\sigma} \cong[\hat{0}, Y]_{\Pi_{d, k}}$. If $d=1$, then $C=\hat{0}$ is the only complement. Thus $\mu_{\sigma}(\hat{0}, C)$ is constant for all $C \leq X$.

Proof. If $d=1$, then since $h^{\sigma}$ is the maximal element, $\hat{0}$ is its only complement. If $d \geq 2$, then we must find a bijection between the elements of $[\hat{0}, C]^{\sigma} \subseteq P^{\sigma}$ for a given $C \in \operatorname{Comp}\left(h^{\sigma}\right)$ in $[\hat{0}, X]^{\sigma}$ and $[\hat{0}, Y] \subseteq \Pi_{d, k}$. Suppose $C$ is as in (8), and assume without loss of generality that $i_{s}=1$ for all $s$. Then for all $l=1, \ldots, j, C \subseteq H_{l, s j+l, r_{s}}$. Given $Z \in[\hat{0}, C]^{\sigma}$, if $Z \nsubseteq H_{\left(\omega_{1}-1\right) i+1,\left(\omega_{2}-1\right) i+1, r}$ for any $r$, then this corresponds to the element $Z^{\prime} \in[\hat{0}, y]$ such that $Z^{\prime} \nsubseteq H_{\omega_{1}, \omega_{2}, r}$ for any $r$. If $Z \subseteq H_{\left(\omega_{1}-1\right) i+1,\left(\omega_{2}-1\right) i+1, r}$, then the corresponding $Z^{\prime} \subseteq H_{\omega_{1}, \omega_{2}, r}$. This correspondence can be defined similarly the other way, $Z^{\prime} \mapsto Z$, so $[\hat{0}, C]^{\sigma} \cong[\hat{0}, Y]$. Thus $\mu_{\sigma}(\hat{0}, C)=\mu_{\Pi_{d, k}}(\hat{0}, Y)$ for all complements $C$ of $h^{\sigma}$ in $[\hat{0}, X]$. 
Lemma 8. Given $X \in \operatorname{Max}\left(P^{\sigma}\right)$, $h^{\sigma}$ has $\left(\frac{n}{d}\right)^{d-1}$ complements in $[\hat{0}, X]^{\sigma}$.

Proof. If $d=1$, then $h^{\sigma}$ is the maximal element of $P^{\sigma}$, so $\hat{0}$ is its only complement. If $d>1$, then for each $s=1,2, \ldots, d-1, i_{s}$, as described in (8), has $n / d$ possible values, all independent of each other. So the number of complements of $h^{\sigma}$ is $\left(\frac{n}{d}\right)^{d-1}$ for $d \geq 1$.

Proof of Theorem 4. Let $C_{X}$ be some complement of $h^{\sigma}$ in $[\hat{0}, X]^{\sigma}$ for each $X \in$ $\operatorname{Max}\left(P^{\sigma}\right)$. Thus:

$$
\begin{aligned}
\sum_{X \in \operatorname{Max}\left(P^{\sigma}\right)} \mu_{\sigma}(\hat{0}, X) & =\sum_{X} \sum_{\begin{array}{c}
C \in \operatorname{Comp}\left(h^{\sigma}\right) \\
\text { in }[\hat{0}, X]^{\sigma}
\end{array}} \mu_{\sigma}(\hat{0}, C) \mu_{\sigma}(C, X) \\
& =\mu(n / d) \sum_{X} \sum_{C} \mu_{\sigma}(\hat{0}, C) \\
& =\mu(n / d)\left(\frac{n}{d}\right)^{d-1} \sum_{X} \mu_{\sigma}\left(\hat{0}, C_{X}\right) \\
& =\mu(n / d)\left(\frac{n}{d}\right)^{d-1} \sum_{Y \in \operatorname{Max}\left(\Pi_{d, k}\right)} \mu_{\Pi_{d, k}}(\hat{0}, Y) \\
& \left.=\mu(n / d)\left(\frac{n}{d}\right)^{d-1}{ }_{(-1)^{d-1}(d-1) !\left(\begin{array}{c}
(k+1) d-1 \\
d-1
\end{array}\right)}^{\quad(\text { Lemmas } 7)} 7 \text { and } 8\right) \\
& =\mu(n / d)\left(-\frac{n}{d}\right)^{d-1}\left(\begin{array}{c}
(k+1) d-1 \\
d-1
\end{array}\right)(d-1) !
\end{aligned}
$$

Equation (9) holds by Crapo's Complementation Theorem. Ordinarily, the sum would be over all $C, C^{\prime} \in \operatorname{Comp}\left(h^{\sigma}\right)$ such that $C \leq C^{\prime}$. But no two complements of $h^{\sigma}$ are comparable, as mentioned right before the statement of this theorem. So the sum is just over all $C \in \operatorname{Comp}\left(h^{\sigma}\right)$.

Equation (10) is true by Lemma 5. Also, $\bigcup_{X}\left\{C \in \operatorname{Comp}\left(h^{\sigma}\right)\right.$ in $\left.[C, X]^{\sigma}\right\}$ has to be a disjoint union. Suppose $C \in \operatorname{Comp}\left(h^{\sigma}\right)$ in both $[\hat{0}, X]^{\sigma}$ and $[\hat{0}, Y]^{\sigma}$ for $X \neq Y$. Then there exist $i$ and $j$ such that $X \subseteq H_{i, j, r_{1}}$ and $Y \subseteq H_{i, j, r_{2}}$, where $r_{1} \neq r_{2}$. If we let $Z=X \wedge Y$, then $i$ and $j$ are in different blocks of $\pi(Z)$, and $Z \nsubseteq H_{i^{\prime}, j^{\prime}, r}$ for any $r$ and for any $i^{\prime}$ in the same block as $i$ of $\pi(Z)$ and any $j^{\prime}$ in the same block as $j$, since $X \subseteq H_{i^{\prime}, j^{\prime}, r_{1}}$ and $Y \subseteq H_{i^{\prime}, j^{\prime}, r_{2}}$. So $Z$ cannot be greater than any complement of $h^{\sigma}$ in $[\hat{0}, X]^{\sigma}$ or in $[\hat{0}, Y]^{\sigma}$. But $C \leq X, Y$, which means $C \leq X \wedge Y=Z$, a contradiction. So it is a disjoint union.

To get the result (11), find $\mu\left(\Pi_{d, k}\right)$ by extracting the coefficient of $t$ in the characteristic polynomial (2). 


\section{A Homology Character from $\mu_{\sigma}\left(P^{\sigma}\right)$}

Again, let $P=\Pi_{n, k}$. Now we define an integer-valued function $\Psi_{n, k}$ on $\mathfrak{S}_{n}$ given by

$$
\Psi_{n, k}(\sigma)=(-1)^{d-1} \mu_{\sigma}\left(P^{\sigma}\right),
$$

where $d$ is the number of cycles of $\sigma$. Note that the cycles do not all have to be the same length; if they are not, then $\mu_{\sigma}\left(P^{\sigma}\right)=0$, so $\Psi_{n, k}(\sigma)=0$.

We now prove that $\Psi_{n, k}(\sigma)$ is, up to a sign, the character afforded by a linear action of $\mathfrak{S}_{n}$ on a suitable homology. In fact, the character is $(-1)^{n-1} \mu_{\sigma}\left(P^{\sigma}\right)$, and $\Psi_{n, k}$ is this character times the sign character of $\mathfrak{S}_{n}$. We use the methods of Stanley in [13].

Let $Q$ be a poset with $\hat{0}$ and $\hat{1}$, and let $\bar{Q}=Q \backslash\{\hat{0}, \hat{1}\}$. We follow the notation of [16]. The order complex $\Delta(\bar{Q})$ is the abstract simplicial complex whose vertex set is $\bar{Q}$ and whose $r$-dimensional faces are all chains of the form $x_{0}<x_{1}<\cdots<x_{r}$ in $\bar{Q}$. The dimension of $\Delta(Q)$ is the largest possible value of $r$ for any chain in $\bar{Q}$.

Now $n$ is the number of elements in the largest chain in $Q$, which means $r \leq n-3$. So for $r=0, \ldots, n-3, C_{r}(\bar{Q})$ is defined to be the vector space over $\mathbb{C}$ whose basis is the $r$-dimensional faces of $\Delta(\bar{Q})$. Also, $C_{-1}(\bar{Q})$ is the one-dimensional vector space generated by the null chain. For all other $r, C_{r}(\bar{Q})=0$. For $r=-1,0, \ldots, n-3$, the map $\partial_{r}: C_{r}(\bar{Q}) \longrightarrow C_{r-1}(\bar{Q})$ is a linear map called the boundary map, defined as

$$
\partial_{r}\left(y_{0}<y_{1}<\cdots<y_{r}\right)=\sum_{i=0}^{r}(-1)^{i}\left(y_{0}<y_{1}<\cdots<\hat{y}_{i}<\cdots<y_{r}\right),
$$

where $\hat{y}_{i}$ means that $y_{i}$ is deleted. The homology of $Q$ for each $r$ is

$$
H_{r}(\bar{Q})=\operatorname{ker} \partial_{r} / \operatorname{im} \partial_{r+1}
$$

Now suppose $P$ is a poset with least element $\hat{0}$ and maybe more than one maximal element. For any $X \in P$, let $Q^{X}=[\hat{0}, X]$. We now define a boundary map the same way as above, except that we include the maximal element $X$ in the chains, following the definition in $[4, \S 5]$. So $\partial_{r}$ defined above corresponds to $\partial_{r+1}$ here. We also define the homology the same way as in (13). The $r$-dimensional homology for the boundary map on the order complex is known as the Whitney homology, denoted $H_{r}^{W}(P)$, which was first defined in [2].

A poset with $\hat{0}$ and $\hat{1}$ is Cohen-Macaulay if every interval $I$ has $H_{r}(I)=0$ whenever $r \neq \operatorname{dim}(\Delta(I))$. As mentioned earlier, $P=\Pi_{n, k}$ is a geometric semilattice. If we add a unique maximal element to the poset $P=\Pi_{n, k}$, then it is a geometric lattice and therefore Cohen-Macaulay by Theorem 4.1 in [6]. So the homology of each maximal interval in $P$ is concentrated in dimension $n-3$. Therefore, by Theorem 5.1 in [4], the Whitney homology of $P$ is

$$
H_{n-2}^{W}(P)=\bigoplus_{X \in \operatorname{Max}(P)} H_{n-3}\left(\bar{Q}^{X}\right)
$$


We use the Lefschetz Fixed Point Theorem (See [13, §1], and it appears that it was first stated in [3]). A version of it states that if the homology of a poset $Q$ is concentrated in a single dimension $r$, then the character of the action of a group $G$ on the homology of $Q$ is $(-1)^{r}$ times the Möbius function of the poset. Note that in many papers, the dimension of the homology of the null chain is 0 . In that case, the character is $(-1)^{r+1}$ times the Möbius function. The sign depends on this.

The action of $\mathfrak{S}_{n}$ on $P$ induces a canonical action on both the homology and the Whitney homology of $P$. For a Cohen-Macaulay poset $Q$, let $\beta^{Q}: \mathfrak{S}_{n} \rightarrow \operatorname{End}(H(Q))$ be the representation with $\mathfrak{S}_{n}$ action on $H(Q)$, the non-trivial top-dimensional homology on $Q$, following the notation of [13]. For any linear representation $\alpha$ of $\mathfrak{S}_{n}$, let $\langle\alpha, \tau\rangle$ be the character of $\alpha$ evaluated at $\tau \in \mathfrak{S}_{n}$. Then here we have

$$
\left\langle\beta^{Q^{X}}, \sigma\right\rangle=(-1)^{n-1} \mu_{\sigma}(\hat{0}, X)
$$

for any $X \in \operatorname{Max}\left(P^{\sigma}\right)$. Therefore, if we let $\beta^{P}$ be the representation with $\mathfrak{S}_{n^{-}}$-action on $H^{W}(P)=H_{n-2}^{W}(P)$, then using (14) and (6),

$$
\begin{aligned}
\left\langle\beta^{P}, \sigma\right\rangle & =\sum_{X \in \operatorname{Max}\left(P^{\sigma}\right)}\left\langle\beta^{Q^{X}}, \sigma\right\rangle \\
& =\sum_{X \in \operatorname{Max}\left(P^{\sigma}\right)}(-1)^{n-1} \mu_{\sigma}(\hat{0}, X)=(-1)^{n-1} \mu_{\sigma}\left(P^{\sigma}\right) .
\end{aligned}
$$

This is the character of the action of $\mathfrak{S}_{n}$ on $H^{W}(P)$. If we combine this with (12), we get $\Psi_{n, k}(\sigma)=(-1)^{n-d}\left\langle\beta^{P}, \sigma\right\rangle$ and hence the following result.

Theorem 9. $\Psi_{n, k}$ is the product of the sign character of $\mathfrak{S}_{n}$ and the character afforded by the action of $\mathfrak{S}_{n}$ on the Whitney homology of $\Pi_{n, k}$.

Note that $(-1)^{n-d}=-1$ only if $n$ is even and $d$ is odd. If $4 \mid n$, then $\mu(n / d)=0$. Therefore, $\Psi_{n, k}$ is the homology character, and is self-conjugate unless $n \equiv 2(\bmod 4)$. (This is an extension of [13, Lemma 7.3].)

\section{$4 \Psi_{n, k}$ is an Induced Character}

Now we know that $\Psi_{n, k}$ is a character, and it is zero for elements of $\mathfrak{S}_{n}$ that are not conjugate to any elements of $\mathfrak{C}_{n}$. So a good direction to go now is to prove that $\Psi_{n, k}$ can be represented as a sum of induced characters $\chi \uparrow_{\mathfrak{C}_{n}}^{\mathfrak{S}_{n}}$, where $\chi$ is an irreducible character of $\mathfrak{C}_{n}$. By [13, Lemma 7.2], $\Psi_{n, 0}$ is simply the value of the induced character $\psi \uparrow_{\mathfrak{C}_{n}}^{\mathfrak{S}_{n}}$, where $\psi$ evaluated at a given $n$-cycle that generates $\mathfrak{C}_{n}$ is $e^{2 \pi i / n}$. We now extend this result to $\Psi_{n, k}$ for $k>0$.

Let $\sigma$ be an $n$-cycle of $\mathfrak{S}_{n}$ in $\mathfrak{C}_{n}$. Let $\zeta=e^{2 \pi i / n}$ and for $s=1, \ldots, n$, let $\chi_{s, n}$ be the character of $\mathfrak{C}_{n}$ such that $\chi_{s, n}(\sigma)=\zeta^{s}$. Then it is known that the $\chi_{s, n}$ are the irreducible 
characters of $\mathfrak{C}_{n}$. Let $\chi_{s, n}^{\star}$ be the induced character $\chi_{s, n} \uparrow_{\mathfrak{C}_{n}}^{\mathfrak{S}_{n}}$. If $\operatorname{gcd}(d, n)=\operatorname{gcd}(s, n)$, then it can be verified that $\chi_{s, n}^{\star}=\chi_{d, n}^{\star}$, so the goal in this section and the next is to prove that

$$
\Psi_{n, k}=\sum_{s \mid n} a_{n, k}^{s} \chi_{s, n}^{\star}
$$

for non-negative integers $a_{n, k}^{s}$. In this section, the main theorem shows that $\Psi_{n, k}$ is an induced character. In the next section, we calculate all the coefficients. It is well-known that if $\zeta$ is a primitive $n$-th root of unity, then

$$
\sum_{i=1}^{n} \zeta^{i r}= \begin{cases}n & \text { if } n \mid r \\ 0 & \text { otherwise }\end{cases}
$$

First, we prove the following lemma.

Lemma 10. The $\chi_{d, n}^{\star}$ for $d \mid n$ are linearly independent.

Proof. For $d \mid n$, let $\lambda_{d, n}=\sum_{i=1}^{n / d} \chi_{d i, n}$. Then $\lambda_{d, n}=1 \uparrow_{\mathfrak{C}_{d}}^{\mathfrak{c}_{n}}$, because if we evaluate it at some $\sigma^{r}$, then we get (16), so $\lambda_{d, n}\left(\sigma^{r}\right)=0$ unless $\frac{n}{d} \mid r$, in which case it is $\frac{n}{d}$. Let

$$
\nu_{d, n}=\sum_{s \mid d} \mu(d / s) s \lambda_{s, n}
$$

Given $r$, let $g=\operatorname{gcd}(n, r)$. Then $\lambda_{s, n}\left(\sigma^{r}\right)=0$ unless $\frac{n}{s} \mid r$, or equivalently, $\frac{n}{g} \mid s$, in which case it is $\frac{n}{s}$. So

$$
\begin{aligned}
\nu_{d, n}\left(\sigma^{r}\right) & =\sum_{s \mid d} \mu(d / s) s \lambda_{s, n}\left(\sigma^{r}\right)=\sum_{s\left|d, \frac{n}{g}\right| s} \mu(d / s) s \lambda_{s, n}\left(\sigma^{r}\right) \\
& =n \sum_{s\left|d, \frac{n}{g}\right| s} \mu(d / s)=n \sum_{s \mid \frac{d g}{n}} \mu(s)= \begin{cases}n & \text { if } g=\frac{n}{d} \\
0 & \text { otherwise }\end{cases}
\end{aligned}
$$

since for any positive integer $m, \sum_{d \mid m} \mu(d)=\delta_{1, m}$. Inducing this up to $\mathfrak{S}_{n}$,

$$
\nu_{d, n}^{\star}\left(\sigma^{r}\right)=\nu_{d, n} \uparrow_{\mathfrak{C}_{n}}^{\mathfrak{S}_{n}}\left(\sigma^{r}\right)= \begin{cases}\frac{n ! \phi(d)}{\mid \operatorname{ccl}_{\mathfrak{S}_{n}\left(\sigma^{r}\right) \mid}} & \text { if } \left.\operatorname{gcd}(r, n)=\frac{n}{d} \text { (or equivalently, } \sigma^{r} \sim \sigma^{n / d}\right) ; \\ 0 & \text { otherwise, }\end{cases}
$$

which, if not zero, is the number of $\tau \in \mathfrak{S}_{n}$ such that $\tau \sigma^{r} \tau^{-1} \in \mathfrak{C}_{n}$. Here, $\operatorname{ccl}_{\mathfrak{S}_{n}}(\sigma)$ is the conjugacy class of $\sigma$ in $\mathfrak{S}_{n}$. From the $\nu_{d, n}^{\star}$, we can get the standard basis of class functions that are zero in all classes that do not contain an element of $\mathfrak{C}_{n}$. Since each $\nu_{d, n}^{\star}$ can be expressed as a linear combination of the $\chi_{d, n}^{\star}$, and vice-versa, and the $\nu_{d, n}^{\star}$ are linearly independent for $d \mid n$, we can conclude that the $\chi_{d, n}^{\star}$ are linearly independent too, since there are the same number of $\chi_{d, n}^{\star}$ as there are $\nu_{d, n}^{\star}$. 
Thus the $\chi_{d, n}^{*}$ for $d \mid n$ are a basis for the induced characters from $\mathfrak{C}_{n}$ up to $\mathfrak{S}_{n}$. By Theorem 3 and the last part of the proof of Lemma 10, $\Psi_{n, k}$ can be expressed as a linear combination, as in (15). We now need to show that the $a_{n, k}^{s}$ are non-negative integers by finding formulas for them. We first determine $a_{n, k}^{n}$, which can be found by simply using inner products. Then we find the $a_{n, k}^{s}$ in the next section.

Lemma 11. $a_{n, k}^{n}=\frac{1}{n} \sum_{d \mid n} \mu\left(\frac{n}{d}\right)\left(\begin{array}{c}(k+1) d-1 \\ d-1\end{array}\right)$ for $n \geq 1$.

Proof. For $n>1$, we show that $a_{n, k}^{n}=\left\langle\Psi_{n, k}, 1_{\mathfrak{S}_{n}}\right\rangle_{\mathfrak{S}_{n}}$. Using Frobenius Reciprocity [12, Th. 1.12.6],

$$
\left\langle\Psi_{n, k}, 1_{\mathfrak{S}_{n}}\right\rangle_{\mathfrak{S}_{n}}=\sum_{d \mid n} a_{n, k}^{d}\left\langle\chi_{d, n}^{\star}, 1_{\mathfrak{S}_{n}}\right\rangle_{\mathfrak{S}_{n}}=\sum_{d \mid n} a_{n, k}^{d}\left\langle\chi_{d, n}, 1_{\mathfrak{C}_{n}}\right\rangle_{\mathfrak{C}_{n}}=a_{n, k}^{n}
$$

The last equality is by orthogonality of irreducible characters. Thus the only time $1_{\mathfrak{S}_{n}}$ appears in the sum (15) is when $d=n$. So for an $n$-cycle $\sigma$,

$$
\begin{aligned}
a_{n, k}^{n} & =\left\langle\Psi_{n, k}, 1_{\mathfrak{S}_{n}}\right\rangle=\frac{1}{n !} \sum_{\tau \in \mathfrak{S}_{n}} \Psi_{n, k}(\tau) \\
& =\frac{1}{n !} \sum_{d \mid n}\left|\operatorname{ccl}_{\mathfrak{S}_{n}}\left(\sigma^{d}\right)\right| \Psi_{n, k}\left(\sigma^{d}\right) \\
& =\frac{1}{n !} \sum_{d \mid n}\left(\frac{n !}{(n / d)^{d} d !}\right) \mu\left(\frac{n}{d}\right)\left(\frac{n}{d}\right)^{d-1}\left(\begin{array}{c}
(k+1) d-1 \\
d-1
\end{array}\right)(d-1) ! \\
& =\frac{1}{n} \sum_{d \mid n} \mu\left(\frac{n}{d}\right)\left(\begin{array}{c}
(k+1) d-1 \\
d-1
\end{array}\right) .
\end{aligned}
$$

For $n=1$, it is clear that $\Psi_{1, k} \equiv 1$ and $a_{1, k}^{1}=1$.

Since $\Psi_{n, k}$ is a character of $\mathfrak{S}_{n}$, it follows by the first assertion in the proof of Lemma 11 that $a_{n, k}^{n}$ is a non-negative integer. For $d \mid n$, let $\mathfrak{C}_{n / d}=\left\langle\sigma^{d}\right\rangle$, and let $\chi_{s, \frac{n}{d}}$ for $s \leq \frac{n}{d}$ be an irreducible character for $\mathfrak{C}_{n / d}$ such that $\chi_{s, \frac{n}{d}}\left(\sigma^{d}\right)=\zeta^{s d}$ for $1 \leq s \leq \frac{n}{d}$. Now let $\chi_{d, n}^{\text {reg }}=\chi_{1, \frac{n}{d}} \uparrow_{\mathfrak{C}_{n / d}}^{\mathfrak{S}_{n}}$. Notice that $\chi_{n, n}^{\text {reg }}$ is the regular character for $\mathfrak{S}_{n}$ and $\chi_{1, n}^{\text {reg }}=\chi_{1, n}^{\star}$. Also, note that the $\chi_{d, n}^{\text {reg }}$ need not be linearly independent. Now we use the next lemma to prove the main result of this section, that $\Psi_{n, k}$ is an induced character from $\mathfrak{C}_{n}$, because each $\chi_{d, n}^{\text {reg }}$ is.

Lemma 12. Let $\sigma$ be an $n$-cycle in $\mathfrak{S}_{n}$. Then we have the following identity:

$$
\frac{1}{d} \chi_{d, n}^{\mathrm{reg}}\left(\sigma^{r}\right)= \begin{cases}\chi_{1, n}^{\star}\left(\sigma^{r}\right) & \text { if } d \mid r \\ 0 & \text { otherwise }\end{cases}
$$


Proof. We know that $\frac{1}{d} \chi_{d, n}^{\mathrm{reg}}\left(\sigma^{r}\right)=\frac{1}{d} \chi_{1, \frac{n}{d}} \uparrow_{\mathfrak{C}_{n / d}}^{\mathfrak{S}_{n}}\left(\sigma^{r}\right)$ and $\chi_{1, n}^{\star}=\chi_{1, n} \uparrow_{\mathfrak{C}_{n}}^{\mathfrak{S}_{n}}$. Since induction of representations is transitive, it is enough to show that

$$
\frac{1}{d} \chi_{1, \frac{n}{d}} \uparrow_{\mathfrak{C}_{n / d}}^{\mathfrak{C}_{n}}\left(\sigma^{r}\right)= \begin{cases}\chi_{1, n}\left(\sigma^{r}\right) & \text { if } d \mid r \\ 0 & \text { otherwise. }\end{cases}
$$

$d \mid r$ if and only if $\sigma^{r} \in \mathfrak{C}_{n / d}$, and then both sides are $\zeta^{r}$. We induce both sides up to $\mathfrak{S}_{n}$, and we are done.

Theorem 13. $\Psi_{n, k}=\sum_{d \mid n} a_{d, k}^{d} \chi_{d, n}^{\mathrm{reg}}$

Proof. Let $\mathfrak{H}_{k, s}=\left(\begin{array}{c}(k+1) s-1 \\ s-1\end{array}\right)$. We know that

$$
\Psi_{n, k}(\tau)= \begin{cases}\mu(d)\left(\frac{n}{d}\right)^{d-1}(d-1) ! \mathfrak{H}_{k, d} & \text { if } \tau \text { is a product of } d \text { cycles } \\ 0 & \text { of length } n / d \text { for some } d \mid n \\ & \text { otherwise }\end{cases}
$$

Again assume that $d \mid n$ and $\sigma$ is an $n$-cycle such that $\mathfrak{C}_{n}=\left\langle\sigma^{d}\right\rangle$.

$$
\begin{aligned}
\sum_{r \mid n} a_{r, k}^{r} \chi_{r, n}^{\mathrm{reg}}\left(\sigma^{d}\right) & =\sum_{r \mid n}\left(\frac{1}{r} \sum_{s \mid r} \mu\left(\frac{r}{s}\right) \mathfrak{H}_{k, s}\right) \chi_{r, n}^{\mathrm{reg}}\left(\sigma^{d}\right) \\
& =\sum_{r \mid n} \frac{1}{r} \chi_{r, n}^{\mathrm{reg}}\left(\sigma^{d}\right) \sum_{s \mid r} \mu\left(\frac{r}{s}\right) \mathfrak{H}_{k, s}=\chi_{1, n}^{\star}\left(\sigma^{d}\right) \sum_{r \mid d} \sum_{s \mid r} \mu\left(\frac{r}{s}\right) \mathfrak{H}_{k, s} \\
& =\chi_{1, n}^{\star}\left(\sigma^{d}\right) \sum_{s \mid d} \mathfrak{H}_{k, s} \sum_{r|d, s| r} \mu\left(\frac{r}{s}\right)=\chi_{1, n}^{\star}\left(\sigma^{d}\right) \sum_{s \mid d} \mathfrak{H}_{k, s} \delta_{d, s} \\
& =\mu(n / d)(n / d)^{d-1}(d-1) ! \mathfrak{H}_{k, d}
\end{aligned}
$$

The second equality of (17) holds because given $s$,

$$
\sum_{r|d, s| r} \mu\left(\frac{r}{s}\right)=\sum_{q \mid \frac{d}{s}} \mu(q)=\delta_{d, s}
$$

For equation (18), [13, Lemma 7.2] proves that $\chi_{1, n}^{\star}\left(\sigma^{d}\right)=\Psi_{n, 0}\left(\sigma^{d}\right)$. Thus we have proved that the right-hand side is equal to a value that we know is $\Psi_{n, k}$. 
Given a real number $x$, let $\langle x\rangle$ be the nearest integer to $x$. Recall that the irreducible characters of $\mathfrak{S}_{n}$ are the $\chi^{\lambda}$ for each partition $\lambda$ of $n$, and $f^{\lambda}$ is the degree of $\chi^{\lambda}$. We now have a way to decompose $\Psi_{p, k}$ into irreducible characters of $\mathfrak{S}_{p}$ for any odd prime $p$. The following corollary is an extension of a result by Stanley.

Corollary 14. Given an odd prime $p$, and a partition $\lambda$ of $p$,

$$
\left\langle\Psi_{p, k}, \chi^{\lambda}\right\rangle_{\mathfrak{S}_{n}}=a_{p, k}^{p} f^{\lambda}+\left\langle f^{\lambda} / p\right\rangle
$$

Proof. By Theorem 13,

$$
\Psi_{p, k}=a_{1, k}^{1} \chi_{1, p}^{*}+a_{p, k}^{p} \chi_{p, p}^{\mathrm{reg}} .
$$

Therefore, since $a_{1, k}^{1}=1$ for all $k$, we get

$$
\left\langle\Psi_{p, k}, \chi^{\lambda}\right\rangle_{\mathfrak{S}_{n}}=a_{p, k}^{p}\left\langle\chi_{p, p}^{\mathrm{reg}}, \chi^{\lambda}\right\rangle+\left\langle\chi_{1, p}^{*}, \chi^{\lambda}\right\rangle=a_{p, k}^{p} f^{\lambda}+\left\langle f^{\lambda} / p\right\rangle
$$

The first summand is well-known about the regular character of $\mathfrak{S}_{n}$ (For example, see Theorem 1.10.1 of [12]). The second summand is by Corollary 7.4 of [13].

\section{The Coefficients $a_{n, k}^{s}$}

By Theorem 13, $\Psi_{n, k}$ is an induced character from $\mathfrak{C}_{n}$ up to $\mathfrak{S}_{n}$. For the sake of completeness, we now determine the coefficients $a_{n, k}^{s}$. This also makes it more clear how to decompose $\Psi_{n, k}$ into irreducibles if $n$ is not prime, since there are references that have the decomposition of induced characters from $\mathfrak{C}_{n}$. See for Example $[15, \S 3]$, where it is done in terms of standard Young tableaux. By the next lemma, each $a_{n, k}^{s}$ is a sum of non-negative integers $a_{d, k}^{d}$ for each $d$ such that $\chi_{d, n}^{\star}$ appears in the expression of $\chi_{s, n}^{\mathrm{reg}}$. All we need to do now is determine what $a_{n, k}^{s}$ is for $s$ properly dividing $n$.

Lemma 15. For $d \mid n, \chi_{d, n}^{\mathrm{reg}}=\sum_{i=0}^{d-1} \chi_{i \frac{n}{d}+1, n}^{\star}$.

Sketch of Proof. It is enough to show that $\chi_{1, \frac{n}{d}} \uparrow_{\mathfrak{C}_{n / d}}^{\mathfrak{C}_{n}}=\sum_{i=0}^{d-1} \chi_{i \frac{n}{d}+1, n}$. Just evaluate both sides at $\sigma^{r}$, and then induce up to $\mathfrak{S}_{n}$.

This shows that for a given $k, a_{n, k}^{s}$ can be expressed in terms of the numbers $a_{d, k}^{d}$. So let $A_{n}^{s}$ be the infinite sequence $\left\{a_{n, k}^{s}\right\}_{k=0}^{\infty}$. Since the expression of $a_{n, k}^{s}$ in terms of the numbers $a_{d, k}^{d}$ is independent of $k$, this is a way to write these numbers so that we do not have to write $k$ all the time. We need to find a formula for these sequences, now that we know the $A_{d}^{d}$ sequences for $d \mid n$. Define the sum of two sequences and scalar multiplication of a sequence the usual way. By theorem $13, a_{d, k}^{d}$ is the coefficient of $\chi_{d, n}^{\text {reg }}$ in $\Psi_{n, k}$, so by Lemma 15 , it appears in the coefficient of $\chi_{(i-1) \frac{n}{d}+1, n}^{\star}$ for $i=1,2, \ldots, d$, 
given $k$. Also, if $s=\operatorname{gcd}(g, n)$ for some $g$, then $\chi_{s, n}^{\star}=\chi_{g, n}^{\star}$, so each $A_{n}^{s}$ sequence can be written as a non-negative integer combination of the $A_{d}^{d}$ sequences for which $a_{d, k}^{d}$ appears in the coefficient of $\chi_{s, n}^{\star}$, and that is for each $d$ such that there exists an $r \equiv 1$ $\left(\bmod \frac{n}{d}\right)$ with $\operatorname{gcd}(r, n)=s$. In this case, $\operatorname{gcd}\left(s, \frac{n}{d}\right)=\operatorname{gcd}\left(r, n, \frac{n}{d}\right)=1$ and $s \mid d$. Our goal is to find how many of these $r$ exist up to modulo $n$, and that will be the coefficient of $A_{d}^{d}$ in $A_{n}^{s}$. First we prove a lemma from number theory that we will use.

Lemma 16. Given positive integers $a, b, t$ such that $\operatorname{gcd}(a, b)=1$, there exists an integer $u$ such that $0 \leq u<t$ and $\operatorname{gcd}(u a+b, t)=1$.

Proof. We form an increasing sequence $a_{0}, a_{1}, \ldots, a_{t}$ of integers that are pairwise relatively prime, or coprime. Let $a_{0}=a$ and $a_{1}=b$. For $m \geq 1$, let

$$
a_{m+1}=a_{m}+a_{0} a_{1} \ldots a_{m-1} .
$$

We prove that these numbers are coprime by induction. First, it is given that $a_{0}$ and $a_{1}$ are relatively prime. Now assuming that $a_{0}, \ldots, a_{m}$ are coprime, we use the Euclidean algorithm to prove that $a_{m+1}$ is relatively prime to all $a_{i}$ for $i=0,1, \ldots, m$. Now $\operatorname{gcd}\left(a_{m}, a_{m+1}\right)=\operatorname{gcd}\left(a_{m}, a_{0} \ldots a_{m-1}\right)$, and for $i<m$,

$$
\operatorname{gcd}\left(a_{i}, a_{m+1}\right)=\operatorname{gcd}\left(a_{i}, a_{m}\right) .
$$

By hypothesis, both are 1, so the numbers in the sequence are all coprime. To prove that the $a_{m}$ are of the form $u^{\prime} a+b$ for some integer $u^{\prime}$ and $m \geq 1$, we know that it holds for $m=1$; it is $0 a+b$. Now assume that it holds for some $m \geq 1$ and prove it for $m+1$. We need to show that $a \mid\left(a_{m+1}-b\right)$. We know that $a_{m+1}-b=a_{m}-b+a_{0} \ldots a_{m-1}$. We also know that $a$ divides $a_{m}-b$ by hypothesis, and $a$ divides $a_{0} \ldots a_{m-1}$ because $a=a_{0}$. This proves that $a_{1}, \ldots, a_{t}$ are $t$ coprime numbers, all congruent to $b$ modulo $a$, and therefore there must be at least one of these numbers that is relatively prime to $t$. Suppose $a_{i}$ is one such number. Let $u^{\prime}=\frac{a_{i}-b}{a}$. In other words, $a_{i}=u^{\prime} a+b$. Then there exists a unique expression $u^{\prime}=u^{\prime \prime} t+u$ such that $0 \leq u<t$. It follows that $\operatorname{gcd}(u a+b, t)=1$.

The number of $r$ up to modulo $n$ such that $r \equiv 0(\bmod s)$ is $n / s$, and the number of such $r$ with $\operatorname{gcd}(r, n)=s$ is $\phi(n / s)$. Now we find how many of these $r$ have $r \equiv 1$ $\left(\bmod \frac{n}{d}\right)$. Let $t_{1}$ and $t_{2}$ be units in the $\operatorname{ring} \mathbb{Z} /\left(\frac{n}{d}\right), t_{1}$ chosen so that there definitely exists an $r$ among these numbers such that $r \equiv t_{1}\left(\bmod \frac{n}{d}\right)\left(t_{1}\right.$ can be $s$, for example). Suppose that $r_{1}, \ldots, r_{w}$ are the numbers $\bmod n$ such that

$$
r_{i} \equiv t\left(\bmod \frac{n}{d}\right) \quad \text { and } \quad \operatorname{gcd}\left(r_{i}, n\right)=s .
$$

for $t=t_{1}$. In $\mathbb{Z} /(n), t_{2}$ is not necessarily a unit, but by Lemma 16 , there exists a $u$ such that $\operatorname{gcd}\left(t_{2}+u\left(\frac{n}{d}\right), n\right)=1$. Then $\left(t_{2}+u\left(\frac{n}{d}\right)\right) r_{1}, \ldots,\left(t_{2}+u\left(\frac{n}{d}\right)\right) r_{w}$ are all congruent 
to $t_{1} t_{2} \bmod n / d$ and distinct $\bmod n$, since the $r_{i}$ were all multiplied by a unit of $\mathbb{Z} /(n)$. This proves that there are at least $w$ of them. To prove there are exactly $w$, consider $t_{2}^{-1}$ in $\mathbb{Z} /\left(\frac{n}{d}\right)$ and suppose there are $w^{\prime}$ numbers, $w^{\prime} \geq w$. By Lemma 16, there exists a $u^{\prime}$ such that $\operatorname{gcd}\left(t_{2}^{-1}+u^{\prime}\left(\frac{n}{d}\right), n\right)=1$. Then the numbers $\left(t_{2}^{-1}+u^{\prime}\left(\frac{n}{d}\right)\right)\left(t_{2}+u\left(\frac{n}{d}\right)\right) r_{i}$ are all congruent to $t_{1} \bmod n / d$, and there are at least $w^{\prime}$ such numbers. Since there are $w$ of them, $w=w^{\prime}$. So we can let $t_{2}=t_{1}^{-1}$, and then we have $w$ numbers that are congruent to $1 \bmod n / d$. Thus for each unit $t \bmod n / d$, there are $w$ numbers up to $\bmod n$ that satisfy (19), so one out of every $\phi(n / d)$ of the numbers $r$ is congruent to $1 \bmod n / d$. Therefore,

$$
A_{n}^{s}=\sum_{d} \frac{\phi(n / s)}{\phi(n / d)} A_{d}^{d},
$$

summing over all $d$ such that $a_{d, k}^{d}$ appears in the coefficient of $\chi_{s, n}^{\star}$.

Example. Suppose $n=72$ and $s=3$. Then the values of $d$ that would have nonzero coefficients in $(20)$ are $d=9,18,36,72$. We do $d=9$ in detail. Let $t_{1}=3$ in $\mathbb{Z} /(8)$. The values of $r$ such that $\operatorname{gcd}(r, 72)=3$ are 3, 15,21,33, 39, 51, 57, 69, and those $r$ such that $r \equiv 3(\bmod 8)$ are 3 and 51 , so $w=2$. Then $t_{1}^{-1} \equiv 3(\bmod 8)$, but 3 is not a unit in $\mathbb{Z} /(72)$. However, by adding $8(u=1)$, we get 11 , which is a unit. So multiply 3 and 51 by 11 to get 33 and 57 , respectively, mod 72 . These are the numbers $r_{i}$ described in (19) for $t=1$. Therefore, the coefficient of $A_{9}^{9}$ in $A_{72}^{3}$ is 2 and $\phi(n / s)=\phi(24)=8$ and $\phi(n / d)=\phi(8)=4$, which verifies (20) here. The expression for $A_{72}^{3}$ in terms of the $A_{d}^{d}$ is

$$
A_{72}^{3}=2 A_{9}^{9}+4 A_{18}^{18}+8 A_{36}^{36}+8 A_{72}^{72} .
$$

There is another equivalent condition on the $d$ that can be used. With this, we will have a quicker way of obtaining which values of $d$ have nonzero terms in the sum. We have the following lemma.

Lemma 17. Given integers $s$ and $d$ that divide $n$, there exists an $r \equiv 1(\bmod d)$ such that $\operatorname{gcd}(r, n)=s$ if and only if $\operatorname{gcd}(s, d)=1$.

Proof. To prove $(\Longrightarrow)$, if such an $r$ exists, then since $s \mid r, \operatorname{gcd}(s, d)=1$.

To prove $(\Longleftarrow)$, if $\operatorname{gcd}(s, d)=1$, then it follows that $s \mid \frac{n}{d}$, so it's enough to show there exists an $r \equiv 1(\bmod d)$ such that $\operatorname{gcd}\left(r, \frac{n}{d}\right)=s$. There exists a $t$ such that $0<t<d$ and $s t \equiv 1(\bmod d)$. This equation also holds if $t$ is replaced by $i d+t$ for $i=0,1, \ldots, \frac{n}{d}-1$. By Lemma $16, \operatorname{gcd}\left(i d+t, \frac{n}{d}\right)=1$ for at least one of these $i$, and then we let $r=s(i d+t)$.

Now in order to find which $d$ have nonzero summands, we only need to find those divisors of $n$ that are relatively prime to $s$, and then divide $n$ by them. Thus we have the result. 
Theorem 18. For $s \mid n$ and some integer $k \geq 0$,

$$
a_{n, k}^{s}=\sum_{\substack{d \mid n \\
\operatorname{gcd}\left(s, \frac{n}{d}\right)=1}} \frac{\phi(n / s)}{\phi(n / d)} a_{d, k}^{d}=\sum_{d} \frac{\phi(n / s)}{d \phi(n / d)} \sum_{t \mid d} \mu\left(\frac{d}{t}\right)\left(\begin{array}{c}
(k+1) t-1 \\
t-1
\end{array}\right) .
$$

\section{Another Proof that $\Psi_{n, k}$ is a Character}

It is also possible to prove that $\Psi_{n, k}$ is a character of $\mathfrak{S}_{n}$ without using homology of posets, as we did in section 3 . In section 4 , the only time the homology result was used was when it was found that $\Psi_{n, k}$ is a character. From this, it was concluded that the $a_{d, k}^{d}$ are all non-negative integers because of Lemma 11. By Theorem 13,

$$
\Psi_{n, k}=\sum_{d \mid n} a_{d, k}^{d} \chi_{d, n}^{\mathrm{reg}}
$$

If we can show that $a_{n, k}^{n}$ is a non-negative integer for each $n$, then we will have shown that $\Psi_{n, k}$ is a sum of characters of $\mathfrak{S}_{n}$, and therefore, a character itself. By Lemma 11,

$$
a_{n, k}^{n}=\frac{1}{n} \sum_{d \mid n} \mu\left(\frac{n}{d}\right)\left(\begin{array}{c}
(k+1) d-1 \\
d-1
\end{array}\right) .
$$

Lemma 19. For positive integers $n$ and $r$, and $k=r-1, a_{n, k}^{n}$ is an integer.

Proof. Let $p$ be a prime divisor of $n$, and let $s$ be the positive integer such that $p^{s} \| n$. Then

$$
\begin{aligned}
n a_{n, k}^{n} & =\sum_{d \mid \frac{n}{p^{s}}}\left(\mu\left(\frac{n}{p^{s-1} d}\right)\left(\begin{array}{c}
p^{s-1} r d-1 \\
p^{s-1} d-1
\end{array}\right)+\mu\left(\frac{n}{p^{s} d}\right)\left(\begin{array}{c}
p^{s} r d-1 \\
p^{s} d-1
\end{array}\right)\right) \\
& =\sum_{d \mid \frac{n}{p^{s}}} \mu\left(\frac{n}{p^{s} d}\right)\left(\left(\begin{array}{c}
p^{s} r d-1 \\
p^{s} d-1
\end{array}\right)-\left(\begin{array}{c}
p^{s-1} r d-1 \\
p^{s-1} d-1
\end{array}\right)\right),
\end{aligned}
$$

since the other summands are definitely zero. We show that each summand is congruent to $0 \bmod p^{s}$.

$$
\begin{aligned}
\left(\begin{array}{c}
p^{s} r d-1 \\
p^{s} d-1
\end{array}\right) & =\frac{\left(p^{s} r d-1\right)\left(p^{s} r d-2\right) \cdots\left(p^{s}(r-1) d+1\right)}{\left(p^{s} d-1\right)\left(p^{s} d-2\right) \cdots 3 \cdot 2 \cdot 1} \\
& =A \frac{\left(p^{s} r d-p\right)\left(p^{s} r d-2 p\right) \cdots\left(p^{s}(r-1) d+p\right)}{\left(p^{s} d-p\right)\left(p^{s} d-2 p\right) \cdots 3 p \cdot 2 p \cdot p} \\
& =A \frac{\left(p^{s-1} r d-1\right)\left(p^{s-1} r d-2\right) \cdots\left(p^{s-1}(r-1) d+1\right)}{\left(p^{s-1} d-1\right) !}=A\left(\begin{array}{c}
p^{s-1} r d-1 \\
p^{s-1} d-1
\end{array}\right)
\end{aligned}
$$


Here, $A$ is a product of fractions of the form $\frac{p^{s} r d-a}{p^{s} d-a}$ for all $a$ relatively prime to $p$. Since the numerator and denominator of each of these fractions are units in $\mathbb{Z} /\left(p^{s}\right)$, all the fractions are equivalent to $1 \bmod p^{s}$. Thus $A \equiv 1\left(\bmod p^{s}\right)$. This proves that

$$
\left(\begin{array}{c}
p^{s} r d-1 \\
p^{s} d-1
\end{array}\right)-\left(\begin{array}{c}
p^{s-1} r d-1 \\
p^{s-1} d-1
\end{array}\right) \equiv 0 \quad\left(\bmod p^{s}\right)
$$

and therefore $n a_{n, k}^{n} \equiv 0\left(\bmod p^{s}\right)$ for each prime divisor $p$ of $n$. Therefore, by the Chinese Remainder Theorem, $n a_{n, k}^{n} \equiv 0(\bmod n)$, and this proves the lemma.

Lemma 20. For $n \geq 2, a_{n, 0}^{n}=0$, and for $k \geq 1, a_{n, k}^{n}>0$.

Proof. We know the case $k=0$. Using [13, Lemma 7.2], $a_{n, 0}^{1}=1$ and $a_{n, 0}^{d}=0$ for $d>1$. For $k \geq 1$, let $r=k+1$. Given a prime divisor $p$ of $n$, let $q_{p}=n / p$. It is well-known that summing over prime divisors of $n, \sum_{p \mid n} \frac{1}{p^{2}}<1$ (This inequality also holds when summed over all primes). So we will prove that

$$
\left(\begin{array}{c}
n r-1 \\
n-1
\end{array}\right)>p^{2}\left(\begin{array}{c}
q_{p} r-1 \\
q_{p}-1
\end{array}\right)
$$

If we let $(a)_{b}=b !\left(\begin{array}{l}a \\ b\end{array}\right)$ for integers $a$ and $b$, then this is equivalent to

$$
(n r-1)_{n-1}>p^{2}(n-1)_{n-q_{p}}\left(q_{p} r-1\right)_{q_{p}-1} .
$$

Since $p q_{p}=n$, it follows that $p^{2}\left(q_{p} r-1\right)\left(q_{p} r-2\right)<(n r-1)(n r-2)$. Thus it is enough to show that

$$
(n r-3)_{n-3}>(n-1)_{n-q_{p}}\left(q_{p} r-3\right)_{q_{p}-3} .
$$

The left side is equal to $(n r-3)_{q_{p}-3}\left(n r-q_{p}\right)_{n-q_{p}}$. It is clear that $n r-3>q_{p} r-3$ and $n r-q_{p}>n-1$ (since $r>1$ ). Thus $(22)$ is true, so for each prime $p \mid n, \frac{1}{p^{2}}\left(\begin{array}{c}n r-1 \\ n-1\end{array}\right)>\left(\begin{array}{c}q_{p} r-1 \\ q_{p}-1\end{array}\right)$. Therefore,

$$
\left(\begin{array}{c}
n r-1 \\
n-1
\end{array}\right)>\left(\sum_{\substack{p \mid n \\
p \text { prime }}} \frac{1}{p^{2}}\right)\left(\begin{array}{c}
n r-1 \\
n-1
\end{array}\right)>\sum_{\substack{p \mid n \\
p \text { prime }}}\left(\begin{array}{c}
q_{p} r-1 \\
q_{p}-1
\end{array}\right)
$$

This inequality holds for each integer $n$. In (21), if one term $\mathfrak{H}_{k, d}$ (cf. proof of Theorem 13) is subtracted, then $\mathfrak{H}_{k, p d}$ is added for some prime $p$, which is a lot larger than $\mathfrak{H}_{k, d}$ by (22). Also, the coefficient of $\mathfrak{H}_{k, n}$ is always 1 . Therefore, $a_{n, k}^{n}>0$.

Thus we have proved the following, which by Theorem 13 proves that $\Psi_{n, k}$ is a character of $\mathfrak{S}_{n}$

Theorem 21. $a_{n, k}^{n}$ is a non-negative integer for all $n \geq 2$, and it is zero only if $k=0$. 
THE ELECTRONIC JOURNAL OF COMBINATORICS 7 (2000), \#R23

\section{Acknowledgments}

I would like to thank my advisor Don Higman and Phil Hanlon for helpful discussions and for encouraging me to send this paper for publication. I would also like to thank the anonymous referee for his comments that helped me improve this paper and make it more understandable.

\section{References}

[1] C. A. Athanasiadis, Characteristic polynomials of subspace arrangements and finite fields, Adv. in Math. 122 (1996), 193-233.

[2] K. Baclawski, Whitney numbers of geometric lattices, Adv. in Math. 16 (1975), $125-138$.

[3] K. Baclawski and A. Björner, Fixed points in partially ordered sets, Adv. in Math. 31 (1979), 263-287.

[4] A. Björner, On the homology of geometric lattices, Algebra Universalis 14 (1982), $107-128$.

[5] H. H. Crapo, The Möbius function of a lattice, J. Combin. Theory 1 (1966), 126131.

[6] J. Folkman, The homology groups of a lattice, J. Math. Mech. 15 (1966), 631-636.

[7] R. Gill, A Generalization of the Partition Lattice: Combinatorial Properties and the Action of the Symmetric Group, Ph.D. thesis, University of Michigan, 1998.

[8] R. Gill, The number of elements in a generalized partition semilattice, Discrete Math. 186 (1998), 125-134.

[9] P. Hanlon, The fixed point partition lattices, Pacific J. Math. 96 (1981), 319-341.

[10] J. Kerr, A basis for the top homology of a generalized partition lattice, J. Algebraic Combin. 9 (1999), 47-60.

[11] G.-C. Rota, On the foundations of combinatorial theory I. Theory of Möbius Functions, Z. Wahrscheinlichkeitstheorie 2 (1964), 340-368.

[12] B. Sagan, The Symmetric Group, Wadsworth and Brooks/Cole, Belmont, CA, 1991.

[13] R. Stanley, Some aspects of groups acting on finite posets, J. Combin. Theory Ser. A 32 (1982), 132-161. 
[14] R. Stanley, Hyperplane arrangements, interval orders, and trees, Proc. Nat. Acad. Sci. U.S.A. 93 (1996), 2620-2625.

[15] J. R. Stembridge, On the eigenvalues of representations of reflection groups and wreath products, Pacific J. Math. 140 (1989), 353-396.

[16] M. L. Wachs, On the (co)homology of the partition lattice and the Free Lie Algebra, Discrete Math. 193 (1998), 287-319.

[17] M. L. Wachs and J. W. Walker, On geometric semilattices, Order 2 (1986), 367385 .

[18] G. M. Ziegler, Matroid shellability, $\beta$-systems, and affine hyperplane arrangements, J. Algebraic Combin. 3 (1992), 283-300. 